\title{
THE ZARISKI PROBLEM FOR FUNCTION FIELDS OF QUADRATIC FORMS
}

\author{
JACK OHM \\ (Communicated by Lance W. Small)
}

\begin{abstract}
By 'a quadratic function field' is meant the affine function field of a nonsingular quadratic form of dimension $>2$. What quadratic function fields contain a given quadratic function field $k(P)$ ? This problem is solved here for quadratic forms $P$ of dimensions 3 and 4 , and an application to the Zariski cancellation problem for quadratic function fields is given.
\end{abstract}

\section{INTRODUCTION}

Let $k$ be a field of characteristic $\neq 2$, and let $P, Q$ be nonsingular quadratic forms of dimension $>2$. By a quadratic function field $k(P) / k$ we shall mean the affine function field of the form $P$. Thus, $\operatorname{dim} k(P) / k=(\operatorname{dim} P)-2$. If $k(P)$ is $k$-isomorphic to a subfield of $k(Q)$, denoted $k(P) \hookrightarrow k(Q)$, then $k(P)$ will be called a quadratic subfield of $k(Q)$ and $k(Q)$ a quadratic overfield of $k(P)$.

0.1 Classical theorem. If $P$ and $Q$ are conics, with $P$ anisotropic, then $k(P) \hookrightarrow$ $k(Q)$ implies $k(P) \cong k(Q)$.

Removing the restriction that $Q$ be a conic (i.e., that $\operatorname{dim} Q=3$ ), we have the

0.2 Generalization. If $P$ is an anisotropic conic, then the quadratic overfields of $k(P)$ are (up to isomorphism) $k(P)$ and $k(P)(t), t$ transcendental over $k(P)$.

Since every (nonsingular) conic is similar (denoted $\approx$ ) to a codimension 1 subform of a 4-dimensional Pfister form, this assertion is, in turn, a corollary to the

0.3 Theorem. Let $P$ be an anisotropic Pfister form of dimension $>2$ and $P^{\prime}$ be a codimension 1 subform of $P$. Then

(1) $k(P)$ is the only quadratic overfield of $k(P)$, and

(2) the quadratic overfields of $k\left(P^{\prime}\right)$ are exactly $k\left(P^{\prime}\right)$ and $k(P)=k\left(P^{\prime}\right)(t)$.

This brings up the general

Quadratic overfield problem. Given a quadratic function field $k(P) / k$, classify (up to $k$-isomorphism) the quadratic overfields of $k(P)$.

Generalization 0.2 solves this problem for $P$ of dimension 3, and the following theorem solves it for $P$ of dimension 4 .

Received by the editors February 14, 1994 and, in revised form, December 9, 1994.

1991 Mathematics Subject Classification. Primary 11E04, 11E81, 12F20.

Key words and phrases. Quadratic form, function field, Zariski problem. 
0.4 Theorem. Let $P$ be an anisotropic 4-dimensional form and $Q$ be a form such that $k(P) ¥ k(Q)$. Then there exists an embedding $k(P) \hookrightarrow k(Q)$ iff there exist $a, b, c \in k^{*}$ with $\sqrt{a b c} \notin k$ such that $P \approx\langle 1, a, b, c\rangle$ and $Q \approx\langle 1, a, b, a b\rangle$. (Furthermore, $k(\langle 1, a, b, a b\rangle)$ is a simple transcendental extension of the function field of the conic $\langle 1, a, b\rangle$.)

A knowledge of the overfields of a given quadratic function field $K$ provides, for that $K$, a solution to the

Quadratic Zariski problem (See $\S 3$ ). Suppose $K / k$ and $K^{\prime} / k$ are quadratic function fields and there exist a finite set of $m$ elements $x$ algebraically independent over $K$ and a finite set of $m$ elements $x^{\prime}$ algebraically independent over $K^{\prime}$ such that $K(x)=K^{\prime}\left(x^{\prime}\right)$. Does it follow that $K \cong K^{\prime}$ ?

The hypothesis of the Zariski problem implies $K \hookrightarrow K^{\prime}$ and $K^{\prime} \hookrightarrow K$ (cf. [O1] or [O2]), hence each field is an algebraic extension of the other, so the quadratic Zariski problem has an affirmative answer in the following cases:

(i) $\operatorname{dim} K / k=1$, by 0.1 .

(ii) $\operatorname{dim} K / k=2$, by 0.4 . (See $\S 3$.)

(iii) $K / k$ is the function field of a Pfister form $P$ of dimension $>2$ or of a codimension 1 subform of $P$, by 0.3 .

(iv) $K / k$ is the function field of a subform of codimension $\leq 4$ of a Pfister form $P$ of dimension $>4$, by (ii) and [AO, Corollary 2.6.1].

(v) $\operatorname{dim} K / k=3$, by (iv) and the Main Theorem of [H1]. (My thanks to Detlev Hoffmann for this observation.)

The tools needed for these results are already implicit in the fundamental works of Knebusch $[\mathrm{K}]$ and, especially, Wadsworth [W], and the proofs given here are for the most part variations on arguments from their papers. Some of the results are apparently known to quadratic forms experts (but to my knowledge they have never been published). For instance, according to the introduction of [H1], 2.2 can be found in the 1974 thesis of D.B. Shapiro.

Notation and terminology. We fix a base field $k$ of characteristic $\neq 2$ and a universal domain $\Omega / k$ (i.e., $\Omega$ is an algebraically closed extension field of $k$ which is of infinite transcendence degree over $k$ ). Thus, 'elements' come from $\Omega$, 'fields' are extensions of $k$ and subfields of $\Omega$, 'isomorphism' means $k$-isomorphism, 'irreducible' means irreducible over $k$, etc. The set of nonzero elements of $k$ is denoted $k^{*}$.

Forms. By 'a form $Q$ of dimension $n$ ' we shall always mean a nonsingular (diagonal) quadratic $k$-form of dimension $n$, i.e., $Q=\left\langle a_{1}, \ldots, a_{n}\right\rangle:=a_{1} X_{1}^{2}+\ldots+a_{n} X_{n}^{2}$, with $a_{i} \in k^{*}$. A form of dimension 3 is called a conic. Two forms are isomorphic (or isometric), $Q \cong Q^{\prime}$, if there exists a nonsingular homogeneous change of variables transforming one into the other; and two forms are similar, $Q \approx Q^{\prime}$, if there exists $a \in k^{*}$ such that $Q \cong a Q^{\prime}$. The coset of $a_{1} \cdots a_{n}$ in $k^{*} /\left(k^{*}\right)^{2}$ (or, by abuse of language, any representative of it) is called the determinant of $Q$ and denoted $\operatorname{det} Q$. If $Q^{\prime}=\left\langle b_{1}, \ldots, b_{m}\right\rangle$, then $Q \perp Q^{\prime}:=\left\langle a_{1}, \ldots, a_{n}, b_{1}, \ldots, b_{m}\right\rangle$, and $Q$ is a subform of a form $P$ if there exists a form $Q^{\prime}$ such that $P \cong Q \perp Q^{\prime}$. The form $Q$ is isotropic if it has a nontrivial $k$-zero, and anisotropic otherwise; and a field $K$ containing a nontrivial $K$-zero of $Q$ is called an isotropy field for $Q$. If there exist $a_{i} \in k^{*}$ such that $Q \cong\left\langle 1, a_{1}\right\rangle \otimes \ldots \otimes\left\langle 1, a_{n}\right\rangle$, then $Q$ is called a Pfister form. A 
form $Q(X)$ represents an element $a \in k^{*}$ if there exists a tuple (b) of elements $b_{i}$ of $k$ such that $Q(b)=a$. A general reference for quadratic forms will be [L].

Function fields. A function field is a finitely generated field extension $K / k$, or sometimes just $K$. We use $\operatorname{dim}$ for the transcendence degree of a function field. A pure transcendental function field is also said to be rational, and we consider the case that $K=k$ as a rational extension of dim 0. A generic zero of an irreducible polynomial $f(X)$ in $n$ indeterminates (over $k$ ) is an $n$-tuple $(x)$ of elements (from $\Omega$ ) such that $f(x)=0$ and $\operatorname{dim} k(x) / k=n-1$. Every irreducible polynomial has a generic zero, which determines a field extension $k(x) / k$ up to $k$-isomorphism; and the function field of $f(X)$ is defined to be this extension $k(x) / k$ (or, more precisely, any element of the isomorphism class of this extension).

The affine function field of, or defined by, an irreducible form $Q\left(X_{0}, X_{1}, \ldots, X_{n}\right)$ is the function field of the dehomogenized polynomial $Q\left(1, X_{1}, \ldots, X_{n}\right.$ ) (which is also irreducible) and is denoted $k(Q)$. The adjective 'affine' will henceforth be dropped, it being understood that by function field of a form we mean its affine function field. (Note: The function field of a form is independent of which indeterminate is used to dehomogenize the form.) Similarly, by a generic zero of $Q$ is meant a generic zero of the dehomogenized polynomial. A function field of a (quadratic) form is also called a quadratic function field for short.

Throughout the paper $P$ and $Q$ will denote forms of dimension $>2$. Such forms are irreducible. Note also that $Q$ is isotropic iff $k(Q) / k$ is rational (e.g. [K, I, p. 72, Proposition 3.8] or [O3, p. 358, Lemma 5.7]), and an anisotropic form remains anisotropic over a rational extension (cf. [L, p. 255, Lemma 1.1]).

\section{Proof of 0.3}

Since $P$ always becomes isotropic over its function field $k(P)$, and therefore also over any field containing $k(P)$, and since $k(P) \hookrightarrow k(Q)$ implies $\operatorname{dim} P \leq \operatorname{dim} Q$, we have the 'only if' direction of the following proposition, which translates the overfield problem into an isotropy problem.

1.1 Theorem. There exists an embedding $k(P) \hookrightarrow k(Q)$ if (and only if) $P$ is isotropic over $k(Q)$ and $\operatorname{dim} Q \geq \operatorname{dim} P$.

Proof. Let $(x)$ be a generic zero of (the dehomogenized) $P$ over $k(Q)$. Then $(x)$ is a fortiori a generic zero of $P$ over $k$, so $k(P)=k(x)$. Since $P$ is isotropic over $k(Q), k(Q)(x) / k(Q)$ is rational. This rationality together with $\operatorname{dim} k(Q) \geq \operatorname{dim} k(x)$ implies $k(x) \hookrightarrow k(Q)$ (cf. [O1] or [O2]).

1.2. Recall that $Q$ is called a Pfister neighbor of a Pfister form $Q^{+}$of dimension $n$ if $Q$ is similar to a subform of $Q^{+}$of dimension $>n / 2$. A Pfister neighbor $Q$ and its associated Pfister form $Q^{+}$have the same isotropy fields ([K, II, pp. 2-3]; so by $1.1 k(Q) \hookrightarrow k\left(Q^{+}\right)$, and if $P$ is isotropic over $k(Q)$ and $\operatorname{dim} P \leq \operatorname{dim} Q^{+}$, then there exists an embedding $k(P) \hookrightarrow k\left(Q^{+}\right)$. For example, by taking $P=\langle 1, a, b, c\rangle$, $Q=\langle 1, a, b\rangle$, and $Q^{+}=\langle 1, a, b, a b\rangle$, we obtain the following corollary, which yields the 'if' portion of 0.4 .

1.3 Corollary. If $a, b, c \in k^{*}$, then $k(\langle 1, a, b, c\rangle) \hookrightarrow k(\langle 1, a, b, a b\rangle)$. 
1.4 Lemma. Two codimension 1 subforms of the same Pfister form are similar.

Proof. Suppose $P^{+}$is the given Pfister form and $Q_{1}, Q_{2}$ are the codimension 1 subforms of $P^{+}$:

$$
P^{+} \cong Q_{1} \perp\left\langle a_{1}\right\rangle \cong Q_{2} \perp\left\langle a_{2}\right\rangle .
$$

Since $P^{+}$is Pfister and $P^{+}$represents $a_{i}, P^{+} \cong a_{i} P^{+}$(cf. [L, p. 279, Corollary 1.7]). Therefore

$$
a_{1} Q_{1} \perp\langle 1\rangle \cong a_{2} Q_{2} \perp\langle 1\rangle .
$$

Hence by Witt cancellation $a_{1} Q_{1} \cong a_{2} Q_{2}$.

1.5 Corollary. If $P$ is a codimension 1 subform of a Pfister form $P^{+}$, then $k\left(P^{+}\right)$ is a simple transcendental extension of $k(P)$.

Proof. Knebusch (cf. [K, I, pp. 72-73] has observed that if $P$ is a 'special' codimension 1 subform of a Pfister form $P^{+}$, then $k\left(P^{+}\right)$is a simple transcendental extension of $k(P)$; and by 1.4 the same is therefore true of every codimension 1 subform of $P^{+}$.

More generally, if $P$ is a Pfister neighbor of codimension $\leq 4$ of a Pfister form $P^{+}$, then $k\left(P^{+}\right)$is ( $k$-isomorphic to) a pure transcendental extension of $k(P)$; cf. [AO, Corollary 2.6].

The proofs of 0.3 and 0.4 hinge on the

1.6 Theorem. Suppose $P$ is anisotropic and similar to a subform of codimension 0 or 1 of a Pfister form $P^{+}$. Then $P$ becomes isotropic over $k(Q)$ (if and) only if either $Q \approx P^{+}$or $Q$ is similar to a subform of $P$.

Proof. If codim $P=0$, i.e. if $P \approx P^{+}$, then $Q$ is similar to a subform of $P^{+}$by $[\mathrm{K}$, I, p. 75, Lemma 4.5] or [W, p. 354, Theorem 2]. If codim $P=1$, then $P$ isotropic over $k(Q)$ implies the same of $P^{+}$; and therefore by the codimension 0 case $Q$ is again similar to a subform of $P^{+}$. Thus, $Q$ is similar to either $P^{+}$itself or to a subform of a codimension 1 subform of $P^{+}$. In the latter case $Q$ is similar to a subform of $P$ by 1.4 .

The next corollary, together with 1.5, gives Theorem 0.3.

1.7 Corollary. Suppose $P$ is anisotropic and $k(P) \hookrightarrow k(Q)$.

(1) If $P$ is similar to a Pfister form, then $Q \approx P$ (hence $k(P) \cong k(Q)$ ).

(2) If $P$ is similar to a codimension 1 subform of a Pfister form $P^{+}$, then either $Q \approx P^{+}$or $Q \approx P\left(\right.$ hence $k(Q) \cong k\left(P^{+}\right)$or $\left.k(Q) \cong k(P)\right)$.

1.8 Corollary. Suppose $k(P) \hookrightarrow k(Q)$ and $\operatorname{dim} P=\operatorname{dim} Q$. Then $P \approx Q$ if either

(1) $P$ is anisotropic and similar to a Pfister form or a codimension 1 subform of a Pfister form, or

(2) $Q$ is anisotropic and similar to a Pfister form or a codimension 1 subform of a Pfister form, and $[k(Q): k(P)]$ is odd.

Proof. The first assertion is by 1.7, and the second may be seen as follows: if $[k(Q): k(P)]$ is odd, then $Q$ is isotropic over $k(Q)$ implies $Q$ is also isotropic over $k(P)$, by Springer's theorem (cf. [L, p. 198, Theorem 2.3]), and then $Q \approx P$ by 1.6 . 
If $Q$ is an anisotropic conic (hence similar to a codimension 1 subform of a Pfister form) and $k(P) \hookrightarrow k(Q)$, then $P$ is anisotropic (equivalently, $Q \approx P$ ) if and only if $[k(Q): k(P)]$ is odd; see $[\mathrm{O} 3, \S 21.5]$ for a computational proof of this result, which also shows that $[k(Q): k(P)]$ can be any odd natural number. Incidentally, I can also give a computational proof of 1.3 which shows that there exists an embedding $k(P) \hookrightarrow k(Q)$ with $[k(Q): k(P)]$ any prescribed even natural number, for the 4-dimensional forms $P=\langle 1, a, b, c\rangle$ and $Q=\langle 1, a, b, a b\rangle$.

\section{Proof of 0.4}

Recall that forms of dimension 4 have function fields of dimension 2. Moreover, if $\operatorname{dim} P=4$, then $P$ is similar to a Pfister form iff $\operatorname{det} P=1$. The main tool in dealing with function fields of 4-dimensional forms is

2.1 Wadsworth's descent theorem ([W, p. 357]). Let $P, Q$ be (nonsingular) forms of dimension 4 with the same determinant $d$. Then $P \approx Q$ if (and only if) $P \approx_{k(\sqrt{d})} Q$.

2.2 Theorem. Let $P$ be an anisotropic form of dimension 4 and $Q$ be a conic. Then $P$ becomes isotropic over $k(Q)$ (if and) only if $Q$ is similar to a subform of $P$.

Proof. Let $Q=\langle 1, a, b\rangle, a, b \in k^{*}$; and let $d=\operatorname{det} P, k^{\prime}=k(\sqrt{d})$, and $c=d / a b$. Then $P$ and $R:=\langle 1, a, b, c\rangle$ both have determinant $d$, and $Q$ is a subform of $R$; so by Wadsworth's descent theorem it suffices to show that $P \approx_{k^{\prime}} R$. This will follow from 1.6, provided we verify that $P$ is similar to an anisotropic Pfister form over $k^{\prime}$ and $P$ becomes isotropic over $k^{\prime}(R)$.

Since $Q$ is irreducible over $k^{\prime}, k^{\prime}(Q)=k(Q)(\sqrt{d})$. Moreover, over $k^{\prime}$ the form $Q$ is a codimension 1 subform of the Pfister form $R$, so by $1.5 k^{\prime}(Q) \hookrightarrow k^{\prime}(R)$. Therefore, $P$ is isotropic over $k^{\prime}(Q)$ implies that $P$ is isotropic over $k^{\prime}(R)$.

Since $\operatorname{det}_{k^{\prime}} P=1, P$ is similar to a Pfister form over $k^{\prime}$; hence it only remains to check that $P$ is anisotropic over $k^{\prime}$, which follows from the

2.3 Lemma. Let $P$ be an anisotropic form of dimension 4 with determinant $d$. Then $P$ remains anisotropic over $k(\sqrt{d})$.

Proof. If $P$ becomes isotropic over $k(\sqrt{d})$, then by [L, p. 200, Lemma 3.1] $P \cong$ $f\langle 1,-d\rangle \perp g\langle 1,-1\rangle$, which implies that $P$ is isotropic, a contradiction.

Remark. I have recently received preprints [H1], [H2], [H3] of some beautiful and penetrating work of Detlev W. Hoffmann (Universität Essen, D-45326, Essen, Germany) on the general question: If an anisotropic form $P$ becomes isotropic over $k(Q)$, under what conditions does it follow that $Q$ is similar to a subform of $P$ ? In particular, he mentions in the introduction to [H1] that Wadsworth has given an example (cf. [L2]) which shows that 2.2 does not generalize to forms of dimension 5, and he proves in [H1, Main Theorem] that the only kind of 5-dimensional counterexample to a generalized 2.2 is a form of the shape $\langle 1, a, b, a b, c\rangle$.

2.4 Corollary (to 2.3). If an anisotropic form $P$ of dimension 4 becomes isotropic over $k(Q)$, then $\operatorname{dim} Q \leq 4$.

Proof. For if we extend the base field to $k^{\prime}:=k(\sqrt{\operatorname{det} P})$, then $P$ is similar to an anisotropic Pfister form over $k^{\prime}$ (by 2.3) which becomes isotropic over $k^{\prime}(Q)$, and therefore by $1.6 \operatorname{dim} Q \leq \operatorname{dim} P$. 
2.5 Corollary. Let $P$ be an anisotropic form of dimension 4, and let $Q=$ $\langle 1, a, b, a b\rangle$, with $a, b \in k^{*}$. Then $P$ becomes isotropic over $k(Q)$ (if and) only if there exists $c \in k^{*}$ such that $P \approx\langle 1, a, b, c\rangle$.

Proof. By $1.5 k(Q)$ is a simple transcendental extension of $k(\langle 1, a, b\rangle)$, so $P$ isotropic over $k(Q)$ implies $P$ isotropic over $k(\langle 1, a, b\rangle)$. But then by $2.2\langle 1, a, b\rangle$ is similar to a subform of $P$, which in turn implies that $P \approx\langle 1, a, b, c\rangle$ for some $c$.

We turn next to the analogue of 2.5 with $\operatorname{det} Q \neq 1$.

2.6 Theorem. Let $P$ be an anisotropic form of dimension 4 and $Q$ be a form of dimension 4, and assume further that $\operatorname{det} Q \neq 1$. Then $P$ becomes isotropic over $k(Q)$ (if and) only if $Q$ is similar to $P$.

Proof. Let $d=\operatorname{det} P$ and $k^{\prime}=k(\sqrt{d})$. Then $P$ is anisotropic over $k^{\prime}(2.3)$ and similar to a Pfister form over $k^{\prime}$, so by $1.6 Q$ is similar to $P$ over $k^{\prime}$. But then $\operatorname{det} Q$ is a square in $k^{\prime}$, and we may assume $k \neq k^{\prime}$. Therefore $\sqrt{d} \notin k$, and by hypothesis $\sqrt{\operatorname{det} Q} \notin k$; so $k(\sqrt{d})=k(\sqrt{\operatorname{det} Q})$, from which it follows that $\operatorname{det} Q=\operatorname{det} P$. Hence $Q \approx P$ by Wadsworth's descent theorem.

The conclusion of 2.6 is false without the $\operatorname{det} Q \neq 1$ hypothesis. For, let $P=$ $\langle 1, a, b, c\rangle$ and $Q=\langle 1, a, b, a b\rangle$, with $c$ a nonsquare. Then $P \not \approx Q$, since their determinants are distinct, but $P$ becomes isotropic over $k(\langle 1, a, b\rangle)$ and therefore also over $k(Q)$ because $k(\langle 1, a, b\rangle) \hookrightarrow k(Q)$.

Note that 2.5 and 2.6 yield Wadsworth's theorem [W] that two anisotropic forms of dimension 4 with the same function field are similar. (The corresponding statement for forms of dimension 5 is false; cf. [AO, Example 1.5].)

2.7 Proof of 0.4. The 'furthermore' assertion of 0.4 has been disposed of in 1.5 and the 'if' assertion in 1.3, so it remains to prove the 'only if' statement.

Note first that $\operatorname{dim} Q=4$ by 2.4. Then by 2.6 and the hypothesis $k(P) \neq k(Q)$, $\operatorname{det} Q=1$; and therefore there exist $a, b, c \in k^{*}$ such that $Q \approx\langle 1, a, b, a b\rangle$. By 2.5 there exists $c \in k^{*}$ such that $P \approx\langle 1, a, b, c\rangle$; and by $1.7 P$ cannot be similar to a Pfister form, so $\operatorname{det} P \neq 1$ and therefore $\sqrt{a b c} \notin k$.

\section{The QUADRATIC ZARISKI PROBlem}

We want to first make explicit the argument for going from 0.4 to an affirmative answer to the quadratic Zariski problem for function fields of 4-dimensional forms. Thus, suppose $K$ and $K^{\prime}$ are function fields of quadric surfaces such that $K(x)=$ $K^{\prime}\left(x^{\prime}\right)$. If $K \cong K^{\prime}$, we are done; so assume $K \not K^{\prime}$. Since $K$ embeds in $K^{\prime}$ and $K^{\prime}$ in $K$ (cf. [O1]), we may further assume that $K / k$ and $K^{\prime} / k$ are not rational. Then by $0.4 K$ and $K^{\prime}$ are function fields of Pfister forms, and by 1.7 we are done.

The hypothesis of the quadratic Zariski problem has some simple reformulations:

3.1 Proposition. Let $P$ and $P^{\prime}$ be forms of the same dimension (>2). Then the following are equivalent:

(1) There exists a finite set $x$ (resp., $\left.x^{\prime}\right)$ of elements algebraically independent over $k(P)$ (resp., $k\left(P^{\prime}\right)$ ) such that $k(P)(x)=k\left(P^{\prime}\right)\left(x^{\prime}\right)$.

(2) There exist embeddings $k(P) \hookrightarrow k\left(P^{\prime}\right)$ and $k\left(P^{\prime}\right) \hookrightarrow k(P)$.

(3) $P$ is isotropic over $k\left(P^{\prime}\right)$ and $P^{\prime}$ is isotropic over $k(P)$.

(4) $P$ and $P^{\prime}$ have the same isotropy fields. 
(5) If $(x)$ is a generic zero of $P$ over $k\left(P^{\prime}\right)$ and $\left(x^{\prime}\right)$ a generic zero of $P^{\prime}$ over $k(x)$, then $k\left(x, x^{\prime}\right)$ is rational over both $k(x)$ and $k\left(x^{\prime}\right)$.

Proof. (1) $\Rightarrow(2)$ by [O1], (2) $\Rightarrow(3)$ because $P$ is isotropic over $k(P),(3) \Rightarrow(4)$ because there exists a place from $k\left(P^{\prime}\right)$ to any isotropy field for $P^{\prime}$ [K, I, p. 69, Theorem 3.3], (4) $\Rightarrow(5)$ because $P$ is isotropic over $k\left(P^{\prime}\right)$ implies $k\left(x, x^{\prime}\right) / k\left(x^{\prime}\right)$ is rational, and $(5) \Rightarrow(1)$ is immediate.

Thus, one reformulation of the problem is: If $P, P^{\prime}$ are anisotropic forms of the same dimension with the same isotropy fields, does it follow that $P$ and $P^{\prime}$ define the same function field? Equivalently, if there exists a place from each function field to the other, does it follow that $k(P) \cong k\left(P^{\prime}\right)$ ? Knebusch [K, I, p. 68] discusses such a notion of 'place equivalence' of quadratic function fields, but he does not go so far as to ask whether it implies isomorphism of the fields for forms of the same dimension. As summarized in $\S 0$, the first open case of this question is for forms $P, P^{\prime}$ of dimension 6 .

Finally, there is a (difficult) counterexample [B] to the Zariski problem for arbitrary (i.e., not necessarily quadratic) function fields. In this example $K$ is the function field of a cubic surface rationally fibered by conics, and there exist elements $x_{1}, x_{2}, x_{3}$ algebraically independent over $K$ such that $K\left(x_{1}, x_{2}, x_{3}\right) / k$ is rational; see [O2] and [O3] for further remarks on the example.

\section{REFERENCES}

[AO] H. Ahmad and J. Ohm, Function fields of Pfister neighbors, J. Algebra 178 (1995), 653-664.

[B] A. Beauville, J.-L. Colliot-Thélène, J.-J. Sansuc, and Sir Peter Swinnerton-Dyer, Varietes stablement rationnelles non rationnelles, Annals of Math. 121 (1986), 283-318. MR 86m:14009

[H1] D. Hoffmann, Isotropy of 5-dimensional Quadratic forms over the function field of a quadric, preprint.

[H2] - On 6-dimensional quadratic forms isotropic over the function field of a quadric, Comm. in Algebra 22(6) (1994), 1999-2014. CMP 94:10

[H3] - Minimal quadratic forms and function fields of quadratic forms, preprint.

[K] M. Knebusch, Generic splitting of quadratic forms I, Proc. London Math. Soc. 33 (1976), 65-93; II Proc. London Math. Soc. 34 (1977), 1-31. MR 55:379

[L] T.Y. Lam, The algebraic theory of quadratic forms, Benjamin, Reading, Mass., 1980. MR 83d:10022

[L2] , Fields of u-invariant 6 after A. Merkurjev, Isarel Math. Conf. Proc., Ring Theory 1989 (in honor of S.A. Amitsur) (L. Rowen, ed.), vol. I, Weizmann Science Press, Jerusalem, 1989, pp. 12-31.

[O1] J. Ohm, On subfields of rational function fields, Arch. Math 42 (1984), 136-138. MR 86h: 12007

[O2] - On ruled fields, Sem. de theorie des nombres, Bordeaux 1, Serie 21 (1989), 27-50. MR 91g:12006

[O3] _ Function fields of conics, a theorem of Amitsur-MacRae, and a problem of Zariski, Algebraic geometry and its applications (C. Bajaj, ed.), Springer-Verlag, 1994, pp. 333-363. MR 95c: 12010

[W] A. Wadsworth, Similarity of quadratic forms and isomorphism of their function fields, Trans. Amer. Math. Soc. 208 (1975), 352-358. MR 51:12702

Department of Mathematics, Louisiana State University, Baton Rouge, Louisiana 70803

E-mail address: mmohm@lsuvax.sncc.lsu.edu 